\title{
De la etnobotánica a los muros verdes: la total relación humano-planta
}

\author{
${ }^{1 *}$ Martha Isabel Torres-Morán, ${ }^{2}$ Blanca Catalina Ramírez-Hernández y ${ }^{3}$ Marisela Rodríguez-Mo- \\ rán
}

From the ethnobotany to the green walls: the total human-plant relationship

${ }^{1}$ Departamento de Producción Agrícola. Centro Universitario de Ciencias Biológicas y Agropecuarias. Camino Ramón Padilla Sánchez No. 2100 Nextipac, Zapopan, Jalisco; '2Departamento de Ecología. Centro Universitario de Ciencias Biológicas y Agropecuarias. Camino Ramón Padilla Sánchez No. 2100 Nextipac, Zapopan, Jalisco; ${ }^{3}$ Departamento de Teorías e Historias. Centro Universitario de Arte Arquitectura y Diseño. Calzada Independencia Norte No. 5075, Huentitán El Bajo S.H. C.P. 44250 Tel. 1202-3000.

*Autor de correspondencia: isabel.torres@academicos.udg.mx

\section{Resumen}

El concepto de relación entre hombre y plantas ha evolucionado tanto como se lo ha permitido el desarrollo tecnológico y el incremento de zonas urbanas. Sin embargo, la humanidad reconoce la dependencia que existe con el entorno vegetal. Desde la incipiente civilización, el hombre ha llevado plantas a su morada y establecido un límite imperceptible entre su necesidad y su voluntad de estar rodeado de ellas. Brindar un entorno bello, no es el único beneficio de las plantas, sin embargo, su efecto positivo debe ser bien ponderado a la luz de la investigación y una nueva educación que afiance el concepto de bienestar que pueden generar. La presente revisión es una mínima contribución en el intento de reconsiderar de nuevo la relación entre el hombre y su entorno vegetal, ya que es necesario tener una visualización interdisciplinaria de ésta.

Palabras clave: Espacios verdes, entorno vegetal, área verde per capita.

\begin{abstract}
Relationship between man and plants has evolved as much as technological development and the increase of urban areas have allowed. However, humanity recognizes how dependence with plant exists. From the beginning of civilization, man has taken plants to his home and established an imperceptible limit between needs and preferences for being surrounded by green. Providing a beautiful environment is not the only benefit of plants, however, its positive effect must be well weighted in the light of research and a new education that strengthens the concept of well being that can generate. The present review is a minimal contribution in the attempt to reconsider again the relationship between man and his plant environment, since it is necessary to have an interdisciplinary visualization of it.
\end{abstract}

Key words: Green spaces, plant environment, green area per capita. 


\section{Introducción}

"El hombre está hecho de plantas", es una afirmación que nadie puede negar pues desde el comienzo de la humanidad, hemos dependido de las plantas para subsistir. Esta relación representa generación tras generación, un continuo movimiento de experiencias y prácticas que modifican la percepción de los pueblos al respecto de la convivencia del humano con su entorno. Las tribus ancestrales y las actuales etnias en todo el mundo han desarrollado sus propias costumbres, cultos religiosos, tabúes, culturas, leyendas y mitos a partir de experiencias con las plantas, puesto que de ellas vienen sus canciones, medicinas, alimentos, vestidos, prácticas y afectos.

El término etnobotánica, denota la relación entre las plantas y el hombre y más allá de la dependencia, las plantas han llamado siempre la atención especial de algunos personajes. Tal es el caso de Alexander von Humboldt (1769 - 1859), él fue, según Manetas (2010), un entusiasta coleccionista de plantas y un experto observador de los efectos del clima y la geografía en las plantas. Igualmente, se puede considerar a Charles Darwin (18091882) y Gregorio Mendel (1822 - 1884) cuyas relaciones con las plantas, determinaron el conocimiento que posteriormente dejaron como legado. Así, en el desarrollo de la historia del mundo y de nuestro país se han suscitado diversas direcciones que relatan diferente modo de relacionarse con las plantas y que han ido delimitando y limitando las fronteras entre las disciplinas que se adentran en el amplio universo vegetal.

En cuanto a lo estrictamente etnobotánico en nuestro país, según Claudine Friedberg, las investigaciones han estado a cargo de biólogos y no de antropólogos y se han enfocado estrechamente a los problemas de gestión del medio ambiente natural (Friedberg, 2013). Esta autora, resume las líneas de investigación etnobotánica en nuestro país en: 1) La evolución del modo de subsistencia de las comunidades campesinas en el marco del desarrollo económico del país, a partir de sus prácticas tradicionales de utilización de plantas silvestres y cultivadas y 2) La salvaguarda del patrimonio de los recursos vegetales naturales y domésticos, en particular desde el punto de vista genético. Sin embargo, en la presente revisión, se presentan evidencias de que las relaciones entre las plantas y el hombre van más allá y han evolucionado también a una velocidad vertiginosa.

Es necesario tener una visualización interdisciplinaria de la relación entre el hombre y su entorno vegetal, ya que se tienen diferentes maneras de interpretar la realidad; según lo analítico, lo afectivo, económico y social, cuya resultante permite una visión que en la actualidad tiene su equilibrio solo en el plano coactivo, es decir en la práctica; de manera que la necesidad de relacionarse con las plantas depende intrínsecamente de la conciencia particular del individuo.

\section{La situación no deseable y la deseable}

Podría decirse que las relaciones entre humanos y plantas pueden ser abordadas desde diferentes puntos de vista. El primer y mas antiguo, es el de producción de alimentos, y es precisamente, el punto de vista que ha generado todas las técnicas actuales de producción, desde el cultivo de granos básicos a cielo abierto y de temporal, hasta la hidroponía que permitió el desarrollo de plantas sin sustrato ${ }^{1}$. Sin embargo, actualmente la necesidad de incrementar la proporción de áreas verdes per capita ${ }^{2}$, ha impulsado por una parte la búsqueda de correlaciones positivas entre plantas y personas y por otra, la

\footnotetext{
${ }^{1}$ La hidroponía como actualmente se conoce, se le debe a Gericke (1938).

${ }^{2}$ La Organización mundial de la Salud recomienda de 9 a $16 \mathrm{~m}^{2}$ de áreas verdes per capita.
} 
investigación para generar nuevas propuestas y a su vez una respuesta en campos que aún son desconocidos.

Las enfermedades relacionadas con el estilo de vida están aumentando dramáticamente en la actualidad. Recientes estudios epidemiológicos en han revelado que la inactividad física representa un alto riesgo de desarrollo prematuro de enfermedades crónicas como las enfermedades cardiovasculares y la diabetes tipo 2 (Rasheed, 2016). Además, hay un marcado aumento en la prevalencia de la obesidad. Actualmente, entre el 30 y el $40 \%$ de los adultos tienen sobrepeso, medido como Índice de Masa Corporal (IMC $>25$ ), y aún más preocupante es el hecho de que casi el 10\% son obesos (IMC> 30). Los problemas asociados con la baja actividad física y el aumento de peso son especialmente alarmantes entre los niños (Hernández, 2016). Además, un estudio reciente ha demostrado que el $44 \%$ de la población experimenta reacciones de estrés con frecuencia en la vida cotidiana. Las enfermedades físicas junto con el estrés mental, la depresión y el síndrome de "agotamiento" se han convertido en un importante desafío económico contemporáneo. Nielsen y Hansen (2006) reportaron un estudio hecho por medio de regresión multinomial en una muestra de 1200 personas en Dinamarca, para comprobar la relación entre el estrés y la obesidad con el acceso de las personas a un jardín o área verde pública (entre otras variables). Sus resultados demostraron que las variables de control que mostraron una correlación estadísticamente significativa con el nivel de estrés fueron: el grupo de edad del encuestado, si tenía un trabajo y si el encuestado tenía un ciclo superior de educación superior. Tener un trabajo o una educación larga en general aumenta las probabilidades de caer dentro de un grupo con un mayor nivel de estrés. El análisis apuntó claramente a las variables de edad como la que

e.cucba / ENERO-JUNIO/ AÑO 4/ NÚMERO 7/25-30 tuvo la correlación más fuerte y persistente con el nivel de estrés experimentado. La correlación con la variable en el modelo de regresión generalmente apunta a que los jóvenes están más estresados y los ancianos menos estresados. Sin embargo, en cuanto al acceso a jardines o áreas verdes, se indicó que mientras más fácil acceso se tiene a áreas de esparcimiento, menor la probabilidad de caer en el grupo más estresado. Por lo tanto, los usuarios frecuentes de áreas verdes rara vez se encuentran entre los más estresados de los encuestados.

Está comprobado el beneficio de las plantas en relación a la disminución del estrés y no solamente referido al contacto con áreas verdes, sino a la misma vegetación que se utiliza como plantas de interior y que puede encontrarse dentro de casas y oficinas.

Las plantas pueden encontrarse en el entorno muy cercano del hombre (Fig. 1). Sin embargo, es cada vez más grande el área dedicada a urbanización, que resulta imprescindible impulsar el incremento de áreas con vegetación, que tengan las características específicas para otorgar beneficios tales como la reducción del estrés. A este respecto, se debe mencionar que las plantas tienen efecto positivo pero intangible en las personas, tal como lo menciona Lohr (2010). Esta autora resalta que se ha comenzado a documentar científicamente este hecho y que existe el fenómeno de que, trabajar en sus jardines, simplemente hace a muchas personas "sentirse bien".

Los efectos tangibles, como la eliminación de contaminantes del aire, el incremento de la humedad relativa, la reducción en la dispersión de partículas en el aire en el interior de una habitación, la reducción de la incidencia de los sonidos se entiende relativamente bien. 
Estos efectos han sido reportados como las características particulares de los muros verdes (Blanc, 2008; Bartesagui et al., 2017). La base de los efectos intangibles, como el aumento de la felicidad, la reducción del estrés y la productividad, no se han comprendido del todo.

En nuestra zona metropolitana, se tienen en algunas zonas con serias carencias de áreas verdes, las cuales se calculan tomando en cuenta todos los espacios que contienen vegetación. Esto puede ser consultado en la página del Sistema de Información Territorial Estatal (SITEL). En esa página puede asociarse la presencia de áreas verdes con los niveles de criminalidad reportados para cada colonia de la ciudad en los noticieros locales. Usualmente, se reconocen niveles mas altos en el índice delictivo, en las

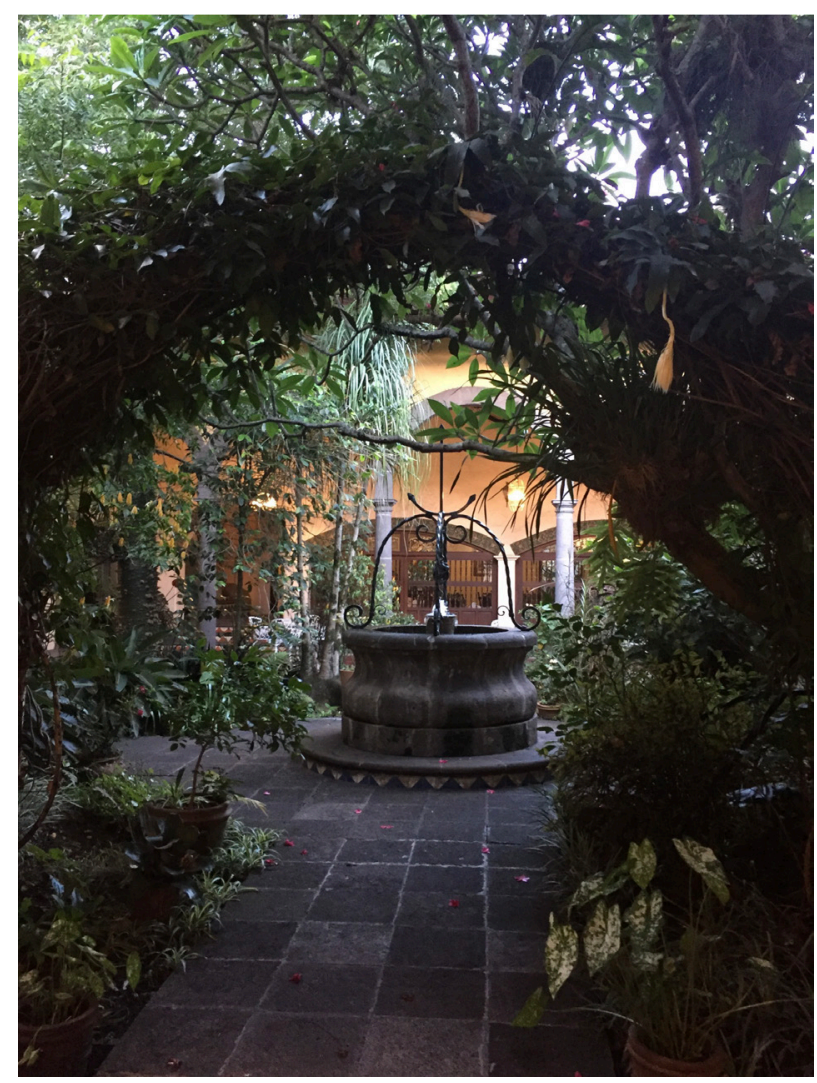

Figura 1. Casa en Coatepec Veracruz, ejemplo del estilo neoclásico íntimamente ligado a la vegetación como parte del diseño arquitectónico de los inmuebles de la época (Siglo XVIII). colonias con menores posibilidades económicas.

Por otra parte, aunque la vegetación ha sido relacionada positivamente con el "miedo a ser asaltado", los residentes que viven en un entorno "más verde" informan niveles más bajos de miedo, menos incivilidades y menos comportamiento agresivo y violento. Esto ha sido reportado en un estudio hecho por (Kuo y Sullivan, 2001) en donde por el contrario de lo que se cree al respecto de la vegetación, proponen que, en algunos entornos, las formas de vegetación que preservan la visibilidad, pueden en realidad disuadir el crimen. Estos autores proponen específicamente, que en lo que ellos llaman "barrios pobres de las zonas urbanas deprimidas" pueden implementarse zonas con vegetación que puede inhibir los niveles delictivos a través de los siguientes mecanismos: aumentando la vigilancia y mitigando algunos de los precursores psicológicos de la violencia. Esto quiere decir, si se incrementa el área de esparcimiento en las colonias, en donde la vegetación sea un elemento importante y abundante, se incrementaría la cantidad de personas que vigilen el entorno, ya que, a más vigilancia, menos delincuencia. $Y$ en el caso de mitigar precursores psicológicos de violencia, se trata de considerar a la vegetación como un elemento importante en la reducción de la llamada "fatiga mental", que, según muchos autores, es la causante de arrebatos de ira y violencia potencial, falta de atención e impulsos de irritabilidad (Kuo y Sullivan, 2001; Lohr, 2011).

Existen también reportes de la relación entre una pronta recuperación de una enfermedad en pacientes que tuvieron acceso a vistas de praderas y zonas con jardines y paisajes arquitectónicamente planeados o bien en hospitales y zonas de recuperación cercanas a áreas con densa vegetación (Ulrich, 1984). 


\section{Retos para el futuro}

La intervención del hombre en los procesos de selección y cultivo de plantas, así como la evolución y adaptación de las mismas, han impactado directamente a la belleza que hoy en día proporcionan a nuestro entorno. Una de las alternativas para reducir la distancia en la relación hombre-planta y acercar el verde en un entorno de exteriores e interiores, son los muros, azoteas y cuadros verdes, que ofrecen la posibilidad de redimensionar el enfoque de la relación hombre-planta a partir de una visión holística que permita integrar un pensamiento nuevo y que éste pueda servir de eje a nuevas investigaciones y una nueva perspectiva integradora.

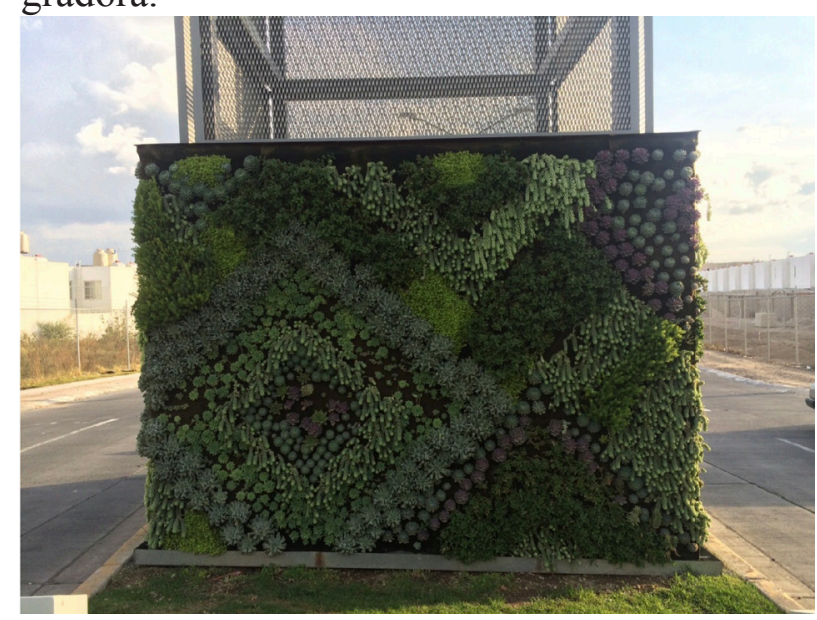

Figura 2. "Cubo verde", realizado por la empresa PLANTIKA con una combinación de plantas de la familia Crassulaceae en la zona de periférico Sur en la ciudad de Guadalajara, Jalisco, México.

La realidad de nuestro entorno es que, las dificultades que se tienen que afrontar hoy en día, rebasan con mucho el quehacer etnobotánico, sin embargo, se pueden aportar elementos muy válidos de análisis y de producción de datos que pueden ser aprovechados en los diferentes ámbitos del quehacer docente, de investigación y de aplicación técnica.

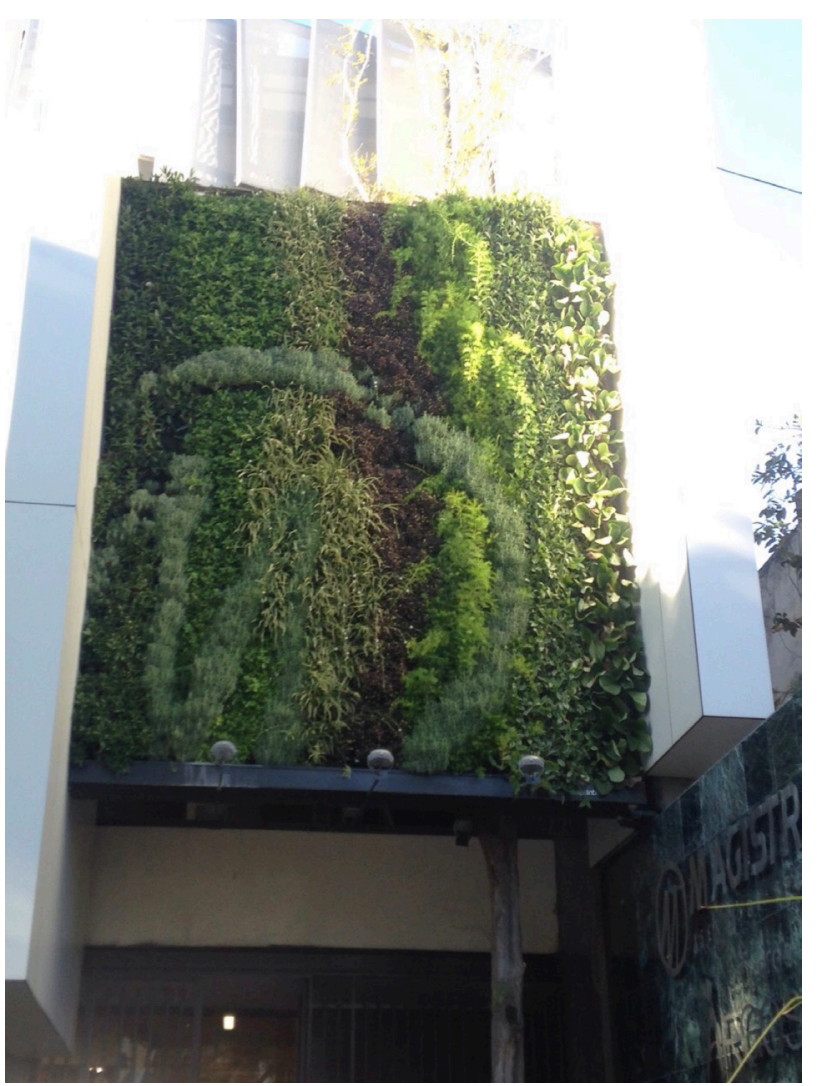

Figura 3. Muro verde realizado por la empresa PLANTIKA con una combinación de plantas de tipo herbáceo en una zona urbana de la ciudad de Guadalajara, Jalisco, México.

De acuerdo a la información presentada, son obvias las necesidades de la población humana que pueden ser solventadas por las plantas, lo cual está llamando la atención a nivel científico y técnico, lo que ha incrementado la investigación enfocada a documentar que el beneficio de las plantas va más allá de las necesidades primarias que satisfacen y de la belleza que proporcionan.

De acuerdo a lo que menciona (Lohr, 2011), la educación también es importante si se quiere cambiar paradigmas de la juventud con respecto a lo que éstas aportan en beneficio de la humanidad. Tanto los estudiantes de carreras relacionadas con plantas, como los que no están relacionados, deben ser educados acerca de estos beneficios para que puedan comprender el valor real y potencial que implica la inversión 
en espacios verdes y que aprendan a comprender también la economía del retorno de esa inversión.

\section{Literatura citada}

Bartesaghi-Koc, C., P. Osmond, and A. Peters. 2017. Towards a comprehensive green infrastructure typology: A systematic review of approaches, methods and typologies. Urban Ecosyst. 20:15-35.

Blanc, P. 2008. The vertical garden: From nature to the city. W.W. Norton, New York, NY.

Friedberg, C., 2013. La etnobotánica Mexicana. Etnobiología. 11(3):813.

Hernández, M. 2016. Encuesta Nacional de Salud y Nutrición de Medio Camino: Resultados ponderados. http://oment.uanl.mx/ wp-content/uploads/2016/12/ensanut_mc_2016-310oct.pdf
La educación actual debe enfocarse a impulsar un cambio de mentalidad, que se verá reflejada en funcionarios de gobierno y líderes que sean capaces de vislumbrar los problemas que se pueden resolver con las plantas.
Kuo, F. y W. Sullivan. 2001. Environment and crime in the inner city Does Vegetation Reduce Crime?. Environment nd Behavior. 33(3): 343-367.

Lohr., V. 2010. What are the benefits of plants indoors and why do we respond positively to them?. Acta Horticulturae. 881(2): 675-682.

Manetas, Y. 2010. Alice in the land of plants. Biology of plants and their importance for planet earth. Springer. pp 385 .

Nielsen, T.N. y K.B. Hansen. 2006. Do access to nature and green areas affect mental stress? Results from a Danish survey on the use of green areas and selected health indicators. Paper for The sixth
European Urban and Regional Studies Conference 21st-24th. citeseerx.ist.psu.edu/viewdoc/ download?doi=10.1.1.542

Rasheed, N., 2016. Prolonged Stress Leads to Serious Health Problems: Preventive Approaches. International Journal of Health Sciences. 10(1): V-VI.

SITEL, http://sitel.jalisco.gob.mx/ portal2/index.php/apps-sicavh

Ulrich, R.S. 1984. Visual landscapes and psychological well-being. Science. 4:17-23. 